\title{
Peter Wahl
}

\section{Was ist wirklich dran an der deutschen Führungsrolle in der EU?}

Dass Deutschland eine Führungsrolle in der Europäischen Union (EU) innehabe, ist so etwas wie Common Sense unter der Mehrheit der Linken. Auf den ersten Blick sprechen auch manche Indizien dafür, dass das so ist:

- Deutschland ist das bevölkerungsreichste Land und die größte Volkswirtschaft der EU;

- die EU-Osterweiterung hat dem deutschen Kapital ungeahnte Verwertungsmöglichkeiten in seinem historischen Hinterhof eröffnet;

- das Europäische Währungssystem ist nach dem Vorbild der Bundesbank konstruiert;

- das Management der Eurokrise folgt über weite Strecken Leitlinien, wie sie von Bundesregierungen aller Couleur vertreten werden und jüngst in Griechenland auch wieder durchgesetzt wurden;

- das internationale Gewicht Deutschlands nimmt seit der Wiedervereinigung kontinuierlich zu - seit dem Jugoslawienkrieg zunehmend mit militärischer Komponente.

Zudem werden von Teilen der Funktionseliten regelmäßig einschlägige Debatten lanciert, so von Bundespräsident Joachim Gauck bei der Münchener Sicherheitskonferenz 2014. Oder es werden spektakuläre Äußerungen vom Stapel gelassen, wie jene von Wolfgang Kauder beim CDUParteitag 2011: „Jetzt auf einmal wird in Europa Deutsch gesprochen." Das alles wird medial unterfüttert - von BILD bis taz und FAZ.

Allerdings ist es in jüngster Zeit um das Narrativ von der deutschen Führung stiller geworden. Auf mehreren für die EU existenziellen Politikfeldern ist von deutscher Führung nicht mehr die Rede:

- in der Flüchtlingskrise ist Deutschland regelrecht isoliert. Nicht nur osteuropäische Länder setzen ihre eigene stockreaktionäre - Linie durch, auch Frankreich, Schweden und Dänemark lassen Merkel im Regen stehen. Die viel beschworene europäische Regelung mit entsprechendem Verteilungsschlüssel wird es nicht geben;

- in den geopolitischen Großkonflikten im Nahen Osten und Afrika und dem damit verbundenen „Krieg gegen den Terror" sind Frankreich und Großbritannien mit ihren Bombern die größeren Spieler, während die Bundeswehr - auch wenn das schon schlimm genug ist - nur rückwärtige Dienste versieht;

- in der bevorstehenden Entscheidung über den BREXIT hat London mit seinem Erpressungspotenzial das Heft des Handelns in der Hand. Ein unterbelichteter Aspekt der Diskussion um den BREXIT ist das Signal, dass Großbritannien nicht bereit ist, sich einer deutschen Führung unterzuordnen;

- und selbst in der Ukraine-Krise, wo Merkel mit MINSK II tatsächlich Führung an den Taggelegt hat, ist Berlin als 
Juniorpartner in die Außenpolitik der Obama-Administration eingebunden. Führung sieht anders aus. Es stellt sich daher die Frage, ob der deutschen Hegemonie ein kurzer Sommer beschieden war oder ob es vielleicht schon von Anfang an damit nicht viel auf sich hatte.

Die Antwort hat beträchtliche politische Konsequenzen. Wenn es stimmt, dass Deutschland Führungsmacht der EU ist, würde der deutschen Linken z.B. auch eine Schlüssel- und Führungsrolle (!) für emanzipatorische Veränderungen zufallen.

\section{Das vertrackte Verhältnis von Nationalstaat - Supranationalität}

Dass die Antwort aufdie „deutsche Frage“ nicht einfach ist, liegt auch in der Sache selbst: Die EU ist ein historisch einzigartiges Hybridgebilde aus einem Staatenbund und Elementen eines Bundesstaates in Entstehung. Daraus ergeben sich komplexe Wechselwirkungen zwischen nationalstaatlicher und supranationaler Ebene. Strukturen, Interessenlagen und Entscheidungsprozesse sind unter Bedingungen der sogenannten Mehrebenen-Governance um Größenordnungen verwickelter als in einem großen Bundesstaat (etwa in den USA). Die Parallelität von selektiver Integration und Souveränitätstransfers einerseits und die Fortexistenz partieller nationalstaatlicher Souveränität andererseits erzeugt oft ein politisches Amalgam, bei dem eine eindeutige Zuordnung zu nationalen Akteuren zwar nicht völlig unmöglich, aber doch kompliziert ist.

Die unübersichtliche Gemengelage führt auch in der linken Debatte immer wieder zu Unschärfe oder sogar logischen Widersprüchen. Ein Beispiel ist der (ansonsten über weite Strecken überzeugende) Beitrag von Michael Heinrich in der PROKLA (180/2015). So heißt es einerseits:
„Die Eurozone wurde auf die deutsche Austeritätspolitik, zu der schon die rotgrüne Koalition einen kräftigen Beitrag geleistet hatte, eingeschworen" (Heinrich 2015: 486). Aber andererseits schreibt Heinrich: „Das von ihnen (Merkel und Schäuble; Anm: P.W.) verfolgte Programm trifft sich durchaus mit den Interessen jener Kapitalfraktionen der anderen EU-Länder, die fit für den Weltmarkt sind und sich der aggressiven deutschen Exportstrategie anschließen wollen." (Ebd.: 487). Im ersten Fall haben wir es mit einer Herrschaftsbeziehung eines dominanten Akteurs $\mathrm{zu}$ anderen zu tun, dem zweiten liegt die Annahme einer Konvergenz von Kapitalinteressen einer ganzen Ländergruppe und die Transnationalisierung - zumindest tendenziell - zugrunde.

Mir scheint an Letzterem viel dran zu sein. Der marxistische, französische Ökonom Claude Serfati beschreibt diese Konstellation jüngst folgendermaßen: „Dominante Staaten und ihr jeweiliges Kapital haben die EU Institutionen geprägt, und sie taten dies in einem historisch ziemlich einzigartigen Kontext: der $\mathrm{Zu}$ sammenbruch und/oder die revolutionäre Herausforderung der Staatsapparate in den meisten europäischen Ländern nach dem Zweiten Weltkrieg, die Notwendigkeit, die europäischen Regierungen zusammenzuführen, um die 'freie Welt' gegen die nach Westen strebenden sowjetischen Kräfte zu schützen und die US-Unterstützung für den Wiederaufbau der kapitalistischen Staaten durch massive Akkumulation, um ihre Dominanz zu sichern. Kurzum, die Analyse der EU-Integration ist ein guter Impfstoff gegen kruden ökonomischen Determinismus." (Serfati 2016: 256f.; Übers.: P.W.)

Mit anderen Worten, in der EU hat es so etwas wie eine hierarchische und partielle Transnationalisierung des Kapitalismus 
gegeben. Natürlich ist die EU deshalb keine basisdemokratische Veranstaltung. Es gibt Herrschaft, „informelle Mechanismen der Macht" (Bourdieu) und eine interne Hackordnung. Deren Spitze jedoch ist umkämpft. Am ehesten kann noch von einem deutsch-französischen Kondominium aber auch das nur mit Einschränkungen - gesprochen werden. Denn auch die Beneluxländer und Italien haben Einfluss, ebenso wie der britische Kapitalismus. Alle anderen stehen dann in der Hierarchie weiter unten. Eine eindeutige, alleinige und stabile Dominanz Frankreichs oder Deutschlands, gibt es jedoch nicht. Vielmehr ergibt sich aus den verschieden nationalen Vektoren ein machtpolitisches Parallelogramm des EU-Kapitalismus. Es handelt sich um einen unabgeschlossenen und ungleichmäßig verlaufenden Prozess, der gegenwärtig in seiner schwersten Krise steckt. Dabei wird zunehmend wieder auf nationale Machtressourcen und partielle Abkopplung von der EU zurückgegriffen. Da wir es also mit einem Mix aus nationalstaatlichen und transnationalen Komponenten zu tun haben, ist eine hegemonietheoretische Analyse, zugegebenermaßen, nicht einfach.

\section{Dominanz, Hegemonie, Führung - oder was?}

Das fängt an mit den Begriffen, mit denen versucht wird, die Sache kategorial zu fassen. Herrschaft, Vorherrschaft, Dominanz, Hegemonie, Führung - alles geht durcheinander.

Auch wenn Definitionen immer diskursiv umkämpft sind, ist es sinnvoll, einige grundlegende Differenzierungen zu beachten:

- Herrschaft bzw. Vorherrschaft beschreibt ein eindeutiges Über- und Unterordnungsverhältnis. Ein Akteur kann einem anderen oder einer Gruppe seinen Willen aufzwingen, ggf. durch militärische Überlegenheit;

- Dominanz ist ebenfalls Herrschaft, klingt aber weniger hart, und wird eher mit dem Einsatz nichtmilitärischer Machtmittel assoziiert;

- Hegemonie wird in den internationalen Beziehungen traditionell als Synonym für Herrschaft gebraucht („die sowjetische Hegemonie über Osteuropa“). Heute wird dem Begriff auch eine systemische Dimension beigemessen, d.h. der Hegemon übernimmt Verantwortung für das internationale System oder Teile davon. Er stellt öffentliche Güter wie Frieden und Stabilität zur Verfügung. Er ist bereit, dafür Eigeninteressen teil- und zeitweise hintanzustellen und höhere Kosten in Kauf zu nehmen. Er ist die internationale Fassung des „guten Fürsten.“ Beliebtes, wenn auch hoch ideologisches Beispiel: die USA und die Pax Americana.

- Im Anschluss an Robert Cox hat die neomarxistische Theoriebildung den Hegemoniebegriff von Gramsci auf das internationale System angewandt (Cox 1983) und dabei neben den Produktionsverhältnissen und dem Staat Ideen (Demokratie, Menschenrechte), Leitbilder (Amercian Way of Life) und kulturelle Momente als Teil von Dominanz definiert.

- Führung (Leadership) wird doppeldeutig benutzt. Einmal als „Initiative ergreifen“, „etwas in die Hand nehmen“, und zwar auf einem zeitlich oder thematisch begrenzten Terrain. So hat Polen in der Visegrad-Gruppe ${ }^{1}$ die Führung bei der Blockade der deutschen Flüchtlingspolitik übernommen. In diesem Sinne, als zeitlich und thematisch be-

1 Polen, Ungarn, Tschechien, Slowakei 
grenzt, wird der Begriff im Folgenden verwendet. Allerdings wird Führung auch synonym zu Hegemonie benutzt: „Amerika muss in der Welt immer die Führung innehaben." (Obama 2014) Zur Realisierung jeder dieser abgestuften Varianten sind die klassischen Machtressourcen notwendig:

- Militärische Macht,

- wirtschaftliches Potenzial, wobei hier nicht allein die quantitativen Dimensionen (Bruttoinlandsprodukt, BIP etc.) eine Rolle spielen, sondern auch qualitative Komponenten, wie technologische Entwicklung und Produktivität, internationale Verflechtungen, Position einer Währung in der Weltwirtschaft etc.,

- politischer Einfluss, durch Diplomatie, in multilateralen Organisationen, Zugehörigkeit zu bestimmten Gremien und Allianzen wie zum Beispiel G7 oder G20, sowie

- kulturelle Attraktivität („soft power“).

Das impliziert, dass jedwede Form von Dominanz, Führung oder Hegemonie eine materielle Basis zur Voraussetzung hat. Selbstverständlich bestehen zwischen den einzelnen Kategorien von Machtressourcen Wechselwirkungen, aus denen sich dann das machtpolitische Gesamtpotenzial eines Landes ergibt.

\section{Deutsche Führung - sektoral und thematisch begrenzt}

Vor dem Hintergrund dieses grob skizzierten Interpretationsrahmens kann man zuerst festhalten, dass die militärische Komponente in den Binnenbeziehungen der EU entfällt. Auch wenn man ansonsten kritische Distanz zur EU hält, die deutschfranzösische Erbfeindschaft ist Geschichte und Krieg im Westen Europas höchst unwahrscheinlich.
Allerdings spielt das Militärische indirekt eine Rolle für den Status innerhalb der EU. Trotz der schleichenden Militarisierung der deutschen Außenpolitik liegen Frankreich und Großbritannien als Atom- und postkoloniale Interventionsmächte hier klar vorn. Nicht zuletzt die Rüstungsausgaben belegen das: Die Deutschen geben 1,2 Prozent ihres BIP für Militär aus, was nicht nur deutlich unter den 2 Prozent liegt, die die NATO fordert, sondern auch fast um die Hälfte unter der Quote Frankreichs und Großbritanniens mit jeweils 2,2 Prozent (SIPRI 2014).

Das wiederholt sich auch politisch im Status von London und Paris als permanente Mitglieder des UN-Sicherheitsrats. Auch wenn Deutschland hier aufholt, etwa durch die Mitwirkung in informellen Gremien wie der Iran-Kontaktgruppe, kann von einer dominanten oder hegemonialen Rolle der deutschen Diplomatie nicht die Rede sein.

Erst recht erledigt sich das Thema kulturelle Attraktivität. Trotz „Willkommenskultur" dürfte außerhalb Bayerns niemand ernsthaft behaupten, dass die Deutschen hier den Franzosen, Briten oder Italienern überlegen wären. Ganz im Gegenteil. Deutschland hat hier mit der Nazivergangenheit - zurecht - ein großes „Handicap.“ Man muss sich nur die Reaktionen aus der Kaczyński-Partei („Recht und Gerechtigkeit“) ansehen, mit der diese auf die Kritik der EU-Exponenten Martin Schulz (SPD) und Günther Oettinger (CDU) an den Maßnahmen gegen das polnische Verfassungsgericht und den öffentlich-rechtlichen Rundfunk antworteten. Berlin ist daraufhin sofort eingeknickt und Regierungssprecher Steffen Seibert formulierte sogar eine kaum verhüllte Distanzierung. In der Sache handelt es sich dabei um einen der seltenen Fälle, in denen Schulz und Oettinger mal keinen 
Unsinn vertreten. Dennoch macht es einen enormen Unterschied, wer so etwas gegenüber wem sagt - und da sehen die Deutschen nun einmal immer wieder alt aus - im wahrsten Sinne des Wortes.

Bliebe also das Potenzial der deutschen Wirtschaft als einzig objektive Basis von Dominanz oder Hegemonie.

\section{Ökonomische Stärke und Hegemonie}

Richtig ist, dass Deutschland die größte Volkswirtschaft der EU ist. Allerdings ist der Abstand zu Frankreich und Großbritannien nicht so groß (ca. 30\%), als dass sich daraus eine wirkliche Überlegenheit ableiten ließe (siehe Tabelle). Wie die aussähe, lässt sich an den Spitzenreitern der Tabelle ablesen.

Hinzu kommt, dass bis zur Mitte des vergangenen Jahrzehnts die deutsche Wirtschaft noch als „kranker Mann“ der Eurozone galt und es ungewiss ist, wie lange sich die aktuelle Position halten lässt. Die exzessive Exportabhängigkeit macht die deutsche Wirtschaft nämlich sehr verwundbar.

\section{Tabelle: BIP ausgewählter Länder 2014 (in Mrd. US-Dollar)}

\begin{tabular}{lr}
\hline USA & 17.419 \\
China & 10.360 \\
Japan & 4.601 \\
Deutschland & 3.852 \\
UK & 2.941 \\
Frankreich & 2.829 \\
Italien & 2.144 \\
\hline
\end{tabular}

Quelle: Weltbank, Online Database. Gross domestic product ranking table. Last Update 18. Sept 2015. URL: http://data.worldbank. org/data-catalog/GDP-ranking-table, Zugriff: 20.12.2015.

Über andere ökonomische Machtressourcen, wie eine eigene Währung, verfügt
Deutschland ebenfalls nicht, ebenso wenig über die Fähigkeit, „Wachstumslokomotive“ bzw. Consumer of Last Resort („Konsument in letzter Instanz") für die Eurozone zu sein - eine Rolle, wie sie China und die USA in den letzten Jahren für die Weltwirtschaft innehatten. Selbst die deutschen Wachstumsraten erinnern eher an den Einäugigen unter Blinden als an einen Motor, der andere mitziehen könnte. Auch qualitative Merkmale wie die überragende Stellung der Londoner City im globalen Finanzkapitalismus kann Deutschland nicht bieten.

Man kann demgegenüber natürlich auch Stärken ins Feld führen, zum Beispiel der relativ hohe Anteil der Industrie an der Wertschöpfung, die Bedeutung der mittelständischen Unternehmen und anderes. Aber aus alledem ergibt sich kein solches Potenzial gegenüber der Konkurrenz, dass daraus eine dominante Position entstehen könnte.

Und last, not least: Selbst in der Eurokrise, wo die deutsche Politik in der Tat Führung übernommen hat, musste Berlin gegenüber der EZB dann doch wieder beträchtliches Terrain aufgeben:

- die diversen Programme zum Aufkauf von Staatsanleihen auf dem Sekundärmarkt sind verkappte Budgetfinanzierung;

- ein inzwischen im Negativbereich angekommener Leitzins ermöglicht es den Krisenländern (aber auch Frankreich und Italien) mit ihren wachsenden Schuldenbergen zinslos und aus deutscher Sicht „disziplinlos" zu leben;

- Mario Draghis berühmte Ankündigung, notfalls grenzenlos Euros zu drucken, macht die EZB de facto zum Lender of Last Resort („Kreditgeber der letzten Instanz").

All das widerspricht diametral dem deutschen Verständnis von Zentralbank. Bei 
Gründung der EZB war die Substanz des Regelsystems (und die Entscheidung über den Sitz in Frankfurt) das Zugeständnis an Berlin, um den Deutschen den Abschied von ihrer heiß geliebten D-Mark zu erleichtern. Draghi bricht jetzt diese Regeln, wenn es ihm notwendig erscheint. In dieser Hinsicht haben seine deutschen KritikerInnen ja Recht. Es ist daher auch kein Zufall, dass schon 2011 Jürgen Stark unter Protest seinen Dienst in der EZB quittierte, ebenso wie 2014 Axel Weber bei der Bundesbank aus gleichen Motiven. Andererseits wäre der Euro wahrscheinlich schon längst kollabiert, wären die Regeln strikt angewendet worden. Damit wird die Zentralbank natürlich nicht zu einem emanzipatorischen Projekt und Draghi nicht zu einem Linken. Die Unabhängigkeit der EZB ist eines der eklatantesten Beispiele für den undemokratischen Charakter der EU. Und in der Sache hat Draghi natürlich nicht das Geringste dagegen, die Subalternen bei jeder Gelegenheit zu kujonieren. Aber er vertritt, im Gegensatz zu Berlin, das Gesamtinteresse der Systemerhaltung.

Deshalb ist die EZB im Krisenmanagement ein machtpolitisch ebenbürtiges Gegengewicht zu Berlin. Dass sie diese Machtposition einnehmen kann, liegt an ihrer „Unabhängigkeit,“ die anders als die der US-Zentralbank Fed, der Bank of England etc. noch einmal um eine Größenordnung „unabhängiger“ ist. Denn als Zentralbank von 18 Ländern ist ja ausdrücklich gewollt, dass keine Regierung Einfluss nehmen kann - zumindest nicht formell.

Merkel schluckt das, nicht zuletzt deshalb, weil eine Alternative nicht in Reichweite ist. Wenn sie zum Management der Eurokrise sagt, sie „fahre auf Sicht“ so ist das durchaus glaubwürdig. Die multiplen Krisen sind auch den Hauptakteuren über den Kopf gewachsen - out of Control! Außer Muddling through bekommen sie nichts mehr auf die Reihe. Hegemonie sieht aber anders aus.

Linke Analysen neigen mitunter dazu, die Herrschenden und ihre Möglichkeiten zu überschätzen. Sie vermuten hinter allem, was geschieht, ausgeklügelte Konzepte und raffinierte Strategien. Demgegenüber scheint ihre Unbeherrschbarkeit geradezu ein Signum der großen Krisen unserer Epoche zu sein - siehe Klimawandel. Das gilt auch für die Widersprüche des Kapitalismus und für die EZB. Sie schafft zwar keine Lösung der Krise, aber sie ermöglicht vorerst noch das, was seit sieben Jahren geschieht: Durchwurschteln.

\section{Profitieren ist nicht führen!}

Viele AutorInnen argumentieren, dass Deutschland Hauptprofiteur der europäischen Integration im Allgemeinen sowie des Binnenmarktes und der Gemeinschaftswährung im Besonderen und zudem Krisengewinnler sei. Auch Frederic Heine und Thomas Sablowski kommen in der PROKLA zu diesem Schluss: „Die deutsche Wirtschaft vermochte es, in allen Phasen der ökonomischen Entwicklung kräftig von der Eurozone zu profitieren“" (Heine/Sablowski 2015: 584).

Das ist vermutlich richtig. Aber: Profitieren bedeutet nicht führen! Deutschland war nicht das einzige Land, das in der Krise gewonnen hat. Seit 2012 sind etwa die britischen Wachstumsraten mehr als doppelt so hoch wie die deutschen. Was immer die Ursachen sind, das ist der übliche Verlauf ungleicher Entwicklung. Mal ist der eine vorn, mal der andere. Erst wenn daraus ein dauerhaftes Muster entsteht und das Profitieren dazu genutzt wird, Machtressourcen aufzubauen, kann eine Verbindung zwischen Profitieren und Führen entstehen. Aber auch dann müssen noch andere Faktoren hinzutreten. Genauso gut ist es aber 
auch denkbar, dass ein Land über einen längeren Zeitraum einfach nur Trittbrettfahrerei betreibt.

\section{Fazit}

Richtig ist: Deutschlands Einfluss in der EU und darüber hinaus hat seit der Wiedervereinigung stetig zugenommen und es ist zu erwarten, dass dies noch eine Weile anhalten wird, nicht zuletzt weil auch die USA in ihrer neuen Globalstrategie („pivot to Asia") die Aktivierung von Juniorpartnern fördern.

Allerdings geht dieser Prozess aufgrund der Souveränitätsbeschränkungen der Nachkriegszeit von einem niedrigen $\mathrm{Ni}$ veau aus. Man könnte von einem Aufholprozess sprechen, um den kontaminierten Begriff von der "Normalisierung" zu vermeiden. Weil Deutschland jetzt vermehrt das tut, was alle anderen schon immer getan haben, nämlich möglichst die eigenen Interessen durchboxen, entsteht der Eindruck eines Aufstiegs zur Führungsmacht.

Perspektivisch sind dem jedoch objektive Grenzen gesetzt, weil Macht primär auf objektiv vorhandenen Ressourcen und nicht auf der Sehnsucht nach Größe beruht. Außerdem würde Hegemonie die Bereitschaft bedeuten, dass Deutschland bereit ist, einen entsprechenden Preis im Interesse der Funktionsfähigkeit der EU oder Eurozone zu zahlen. Tatsächlich ist das Gegenteil der Fall.

Insofern ist das Narrativ von der deutschen Dominanz oder gar Hegemonie in der EU völlig überzogen. Angetrieben wird es von einer Fraktion der Funktionseliten, die - wie ihre deutschnationalen Vorgänger - dazu tendiert, Wunsch und Wirklichkeit zu verwechseln, und die zu Selbstüberschätzung, wenn nicht Größenwahn neigt.

Demgegenüber braucht die Linke eine autonome und realistische Analyse der
Möglichkeiten und Grenzen deutscher Macht. Der antinationale Impetus der deutschen Linken ist eine Errungenschaft, die es unbedingt zu bewahren gilt. Insofern heißt es, weiterhin allen Versuchen der deutschen Funktionseliten entgegenzutreten, sich wieder zur Großmacht aufzuschwingen - innerhalb der EU wie außerhalb. Aber wir sollten uns auch nicht von subjektiven Wünschen blenden lassen. Hegemonie ist keine Sache von Wollen, sondern von Können. Warum sollte die Linke, die einem Gauck oder Kauder doch auch sonst nicht mal ein gebrauchtes Moped abkaufen würde, auf deren Großmachtfantasien hereinfallen?

\section{Literatur}

Cox, Robert W. (1983): Gramsci, Hegemony and International Relations: An Essay in Method. In: Millennium. Journal of International Studies 12(2): 162-175.

Heine, Frederic/Sablowski, Thomas (2015): Zerfällt die Europäische Währungsunion? Handels- und Kapitalverflechtungen, Krisenursachen und Entwicklungsperspektiven der Eurozone. In: PROKLA 45(4): 563-591.

Heinrich, Michael (2015): Gute deutsche Politik. In: PROKLA 45(3): 483-489.

Obama, Barack (2014): Remarks by the President at the United States Military Academy Commencement Ceremony. May 28, 2014. URL: whitehouse.gov/the-pressoffice/2014/05/28/remarks-president-westpoint-academy-commencement-ceremony, Zugriff: 20.12.2015.

Serfati, Claude (2016): EU Integration as Uneven and Combined Development. In: Research in Political Economy 30B: 255-294.

SIPRI, Stockholm Peace Research Institute (2014): SIPRI Military expenditure database. URL: sipri.org/research/armaments/ milex/milex_database,_Zugriff: 20.12.2015. 